\title{
Onivyde for the therapy of multiple solid tumors
}

in the following Dove Press journal:

OncoTargets and Therapy

20 May 2016

Number of times this article has been viewed

\author{
Haijun Zhang \\ Department of Oncology, Zhongda \\ Hospital, Medical School, Southeast \\ University, Nanjing, People's Republic \\ of China
}

\begin{abstract}
Drug delivery system based on nanobiotechnology can improve the pharmacokinetics and therapeutic index of chemotherapeutic agents, which has revolutionized tumor therapy. Onivyde, also known as MM-398 or PEP02, is a nanoliposomal formulation of irinotecan which has demonstrated encouraging anticancer activity across a broad range of malignancies, including pancreatic cancer, esophago-gastric cancer, and colorectal cancer. This up-to-date review not only focuses on the structure, pharmacokinetics, and pharmacogenetics of Onivyde but also summarizes clinical trials and recommends Onivyde for patients with advanced solid tumors. Keywords: irinotecan, PEP02, MM-398, Onivyde, drug delivery system, tumor therapy
\end{abstract}

\section{Introduction}

Irinotecan (CPT-11) is a semisynthetic derivative of camptothecin which targets topoisomerase I, and is currently used in the treatment of multiple solid tumors, such as metastatic colorectal cancer (mCRC), small-cell lung cancer, non-small-cell lung cancer, gastric cancer, and cervical cancer. ${ }^{1-4}$ The pharmacology of irinotecan is complicated, with extensive metabolic conversions involved in the activation, inactivation, and elimination. ${ }^{5-7}$ Irinotecan as a prodrug is converted by nonspecific carboxylesterases into SN-38, which is a 100- to 1,000-fold more active metabolite. . $^{7}$ SN-38 is glucurono-conjugated in the liver, and this metabolite, although inactive, may participate in the enterohepatic cycling of SN-38 after hydrolysis in the intestinal lumen. ${ }^{9}$ The SN-38 metabolite is cleared by the biliary route after glucuronidation by uridine diphosphate-glucuronosyltransferase 1A1 (UGT1A1). UGT1A1 activity exhibits a wide intersubject variability, in part related to UGT1A1 gene polymorphisms. Patients homozygous for the UGT1A1*28 allele are at increased risk of developing hematological and/or digestive toxicities. ${ }^{10}$ However, hydrolysis of active lactone rings in irinotecan and SN-38 to inactive carboxylate forms occurs in normal physiologic $\mathrm{pH} .{ }^{11}$ The clinical disadvantage of irinotecan includes acute toxicities, fast elimination of the drug, and risk of diarrhea and neutropenia as the major dose-limiting toxicities (DLTs). ${ }^{7,8}$ These properties of the drug contribute to the limitation of its clinical application. Hence, the rationally designed drug delivery system (DDS) represents a strategy to improve the pharmacokinetics and biodistribution of irinotecan while protecting it from premature metabolism and extending the time of its exposure at the site of action. Advances in nanotechnology have revolutionized medicines, especially for tumor therapy. Nano-DDS is capable of passive drug delivery through the enhanced permeability and retention (EPR) effect or active targeting via binding to receptors associated with the tumor, thus improving the pharmacokinetics and therapeutic index of chemotherapeutic agents. ${ }^{12-16}$ Due to the perfect biocompatibility, liposomebased drug carrier has become a favorable DDS for various purposes. ${ }^{7,8}$ For example, liposomal doxorubicin, and particularly pegylated liposomal doxorubicin, has shown
Correspondence: Haijun Zhang Department of Oncology, Zhongda Hospital, Medical School, Southeast University, 87 Dingjiaqiao, Nanjing 210009, People's Republic of China $\mathrm{Tel} / \mathrm{fax}+862583275416$ Email zhanghaijunseu@।63.com (c) (i) (5) 2016 Zhang. This work is published and licensed by Dove Medical Press Limited. The full terms of this license are available at https://www.dovepress.com/tterms.php cc) ${ }_{\mathrm{BY}} \mathrm{NC}$ and incorporate the Creative Commons Attribution - Non Commercial (unported, v3.0) License (http://creativecommons.org/licenses/by-n/3.0/). By accessing the work you hereby accept the Terms. Non-commercial uses of the work are permitted without any further permission from Dove Medical Press Limited, provided the work is properly attributed. For permission for commercial use of this work, please see paragraphs 4.2 and 5 of our Terms (https://www.dovepress.com/terms.php). 
significant pharmacologic advantages and an added clinical value over doxorubicin. ${ }^{17}$

Onivyde, also known as MM-398 or PEP02, has been designed and developed as a nanoliposomal formulation of irinotecan, which improves the pharmacokinetics of the drug by increasing drug encapsulation and loading efficiency, protecting the drug in the active lactone configuration, prolonging circulation time, providing sustained release, rerouting the drug from sites of toxicity such as the gastrointestinal tract, increasing tumor accumulation via the EPR effect, and reducing host toxicity. ${ }^{7,8}$ Based on the encouraging preclinical and clinical data available for the treatment of a variety of solid tumors, Onivyde was newly approved by the US Food and Drug Administration (FDA) in October 2015 as a combination regimen for patients with gemcitabinebased chemotherapy-resistant metastatic pancreatic cancer. In addition, it is also currently undergoing Phase II/III clinical trials for the therapy of many cancer types, such as pancreatic cancer, esophago-gastric (OG) cancer, and colorectal cancer. ${ }^{18-21}$ These clinical trials demonstrate that Onivyde has potential antitumor activity across a broad range of advanced solid tumors. ${ }^{7,8,18-21}$

In this review, the structure, pharmacokinetics, and pharmacogenetics of Onivyde are addressed. Clinical trials are also summarized, and recommendations are made for
Onivyde for patients with advanced pancreatic cancer and other cancer types.

\section{Structure}

Molecular formula of irinotecan is C33H38N4O6, and chemical formula is 7-ethyl-10-[4-(1-piperidino)-1-piperidino] carbonyloxycampothecin, which is shown in Figure 1A. Onivyde, the nanoliposomal formulation of irinotecan, employs a modified gradient-loading method using sucrose octasulfate with unprecedented drug-loading efficiency and in vivo drug stability. ${ }^{7}$ Figure $1 \mathrm{~B}$ schematically illustrates the structure of Onivyde.

\section{Pharmacokinetics}

The pharmacokinetics of nanoliposomal CPT-11 formulated using either TEA-SOS or TEA-Pn was determined in normal female rats, which is summarized in Table $1 .^{7}$ Nanoliposomal CPT-11 was associated with significantly longer circulation times and less rapid clearance from the blood than free CPT-11. What is more, to define pharmacokinetics of Onivyde in human, a total of eleven patients were randomized into three dose levels - 60 (one patient), 120 (six patients), and $180 \mathrm{mg} / \mathrm{m}^{2}$ (four patients) - in a study by Chang et al. ${ }^{22}$ The formulation of Onivyde may modify the pharmacokinetic parameters of irinotecan and SN-38 by
A

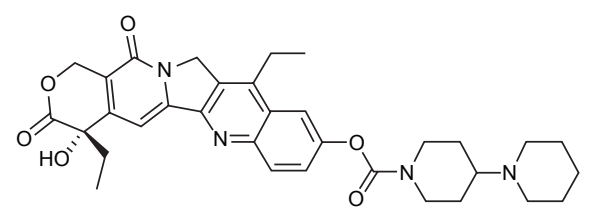

B

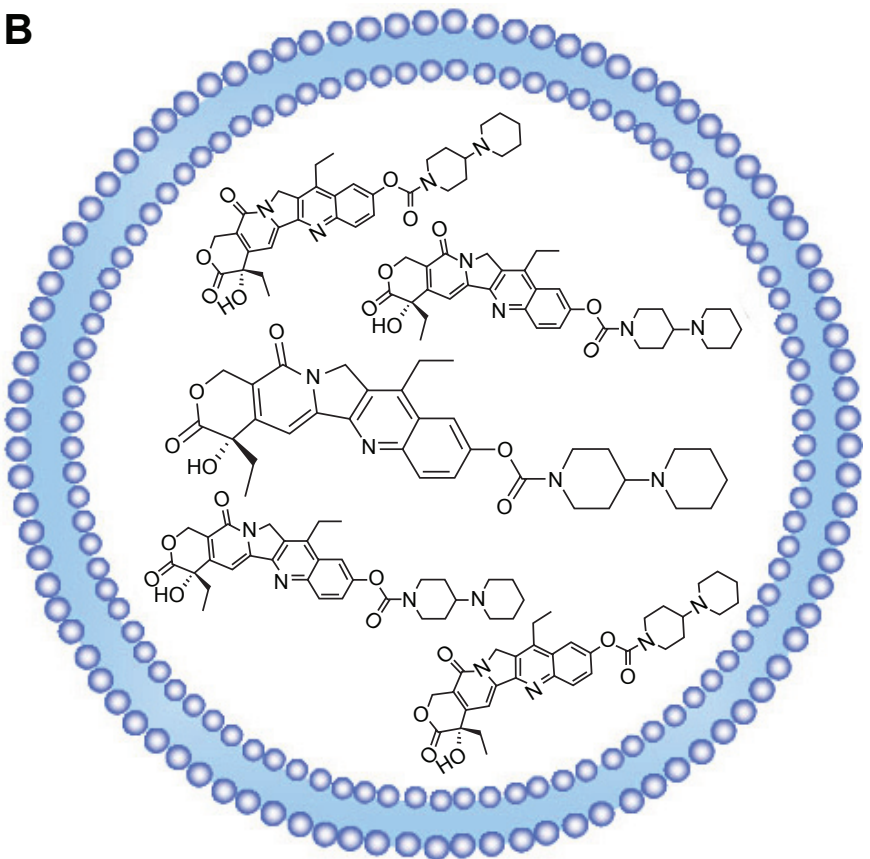

Figure I Chemical structure of irinotecan (A). Schematic structure of Onivyde: the basic molecule irinotecan forms a nanoscale complex with either poly(phosphate) or sucrose octasulfate in the liposome interior (B). 
Table I Pharmacokinetic variables for free and nanoliposomal CPT-II in rats

\begin{tabular}{lll}
\hline Pharmacokinetic variables & $\begin{array}{l}\text { Free } \\
\text { CPT-I I }\end{array}$ & $\begin{array}{l}\text { Nanoliposomal } \\
\text { CPT- I I }\end{array}$ \\
\hline $\mathrm{t}_{1 / 2}$ (hours) & 0.27 & 6.80 \\
AUC $_{\infty}(\mu \mathrm{g} \cdot \mathrm{h} / \mathrm{mL})$ & 6.2 & $1,407.8$ \\
$\mathrm{CL}(\mathrm{mL} / \mathrm{h})$ & 1,609 & 7.10 \\
$\mathrm{~V}_{\mathrm{d}}(\mathrm{mL})$ & 616.4 & 69.7 \\
MRT (hours) & 0.4 & 9.8 \\
$\mathrm{t}_{1 / 2}$ of CPT-II release (hours) & - & 14.0 \\
\hline
\end{tabular}

Abbreviations: $\mathrm{t}_{1 / 2}$, terminal half-life; $\mathrm{AUC} \mathrm{C}_{\infty}$, area under the concentration vs time curve in plasma based on the sum of exponential terms; $\mathrm{CL}$, clearance calculated from exponential terms; $V_{d}$, volume of distribution; MRT, mean residence time calculated from exponential terms.

liposomal encapsulation. Comparing with the pharmacokinetics after free irinotecan injection, the dose-normalized pharmacokinetics of SN-38 following Onivyde administration was characterized by lower maximum plasma concentration $\left(\mathrm{C}_{\max }\right)$, prolonged terminal half-life $\left(\mathrm{t}_{1 / 2}\right)$, and higher area under curve (AUC), although with significant interindividual variation. The $\mathrm{C}_{\max }$, terminal $\mathrm{t}_{1 / 2}$, and $\mathrm{AUC}$ of $\mathrm{SN}-38$ after administration of $120 \mathrm{mg} / \mathrm{m}^{2}$ of Onivyde were $9.2 \pm 3.5 \mathrm{ng} / \mathrm{mL}$, $75.4 \pm 43.8$ hours, and $710 \pm 395 \mathrm{ng} \cdot \mathrm{h} / \mathrm{mL}$, respectively. Slow clearance is also an advantage of Onivyde. ${ }^{23}$ The levels of CPT-11 and SN-38 following Onivyde administration were persistent and remained in circulation for over 50 hours, whereas the CPT-11 and SN-38 in the plasma were cleared rapidly from circulation within 8 hours after free irinotecan injection.

\section{Pharmacogenetics}

Genetic polymorphisms affect toxicity and efficacy of irinotecan, and a pharmacogenetic study showed that a patient with a combined heterozygous genotype of UGT1A $1 * 6 / * 28$ had significantly higher $\mathrm{C}_{\max }$ and AUC levels of SN-38 after Onivyde administration. ${ }^{22}$ In another study, in the group administered with Onivyde, the frequency of grade 3-4 neutropenia was higher among UGT1A $1 * 6$ heterozygotes compared with those with wild-type genotype (40\% [two out of five] for heterozygotes vs 3\% [one out of 30] for wild type, $P=0.0220) .{ }^{18}$ Patients with combined heterozygous genotype of UGT1A1*6/*28 had severe toxicity leading to death.

\section{Preclinical research}

In a series of preclinical studies, Onivyde demonstrated significantly superior anticancer efficacy when compared to free irinotecan in multiple cell lines and xenograft models, including colorectal, gastric, breast, cervical, pancreatic, and lung cancer and glioma, as well as Ewing's sarcoma family of tumors. ${ }^{7,823}$ At the equivalent dose, free irinotecan showed massive tumor progression, while Onivyde showed enhanced antitumor efficacy. ${ }^{7,823}$ The striking antitumor activity in the majority of xenograft models showed with Onivyde was significantly correlated with its advantageous pharmacokinetic properties, by which Onivyde achieved higher intratumoral levels of the prodrug irinotecan and its active metabolite $\mathrm{SN}-38$ compared with free irinotecan. Thus, the enhanced accumulation of the active metabolite within the tumor microenvironment could safely improve its antitumor activity with low systemic toxicity.

\section{Clinical trials of Onivyde for the therapy of multiple solid tumors}

The efficacy of Onivyde for the therapy of a variety of solid tumors has been investigated in several clinical trials, including various Phase I, II, and III studies on registry, which are summarized in Table 2.

\section{Phase I}

In the first-in-human Phase I trial, eleven patients with advanced refractory solid tumors were enrolled to determine the maximum tolerated dose (MTD), DLT, safety

Table 2 Characteristics of the clinical trials of Onivyde for the treatment of multiple solid tumors

\begin{tabular}{|c|c|c|c|c|}
\hline Study & Trial & Design & Tumor type & Outcomes \\
\hline Chang et $\mathrm{a}^{22}$ & Phase I & Dose escalation & Advanced solid tumors & MTD: $120 \mathrm{mg} / \mathrm{m}^{2}$ monotherapy at 3-week interval \\
\hline Roy et $\mathrm{al}^{18}$ & Phase II & Randomized, three-arm & Esophago-gastric cancer & ORR: I $3.6 \%$ (Onivyde) vs $6.8 \%$ (irinotecan) vs I5.9\% (docetaxel) \\
\hline Chibaudel et al ${ }^{19}$ & Phase II & $\begin{array}{l}\text { Randomized, } \\
\text { noncomparative }\end{array}$ & Colorectal cancer & ORR: $16.7 \%$ (Onivyde + 5-FU/LV) vs $11.5 \%$ (irinotecan + 5-FU/LV) \\
\hline Ko et $\mathrm{a}^{20}$ & Phase II & $\begin{array}{l}\text { Multicenter, } \\
\text { open-label, single arm }\end{array}$ & Pancreatic cancer & mOS: 5.2 months; mPFS: 2.4 months \\
\hline Von Hoff et $\mathrm{al}^{21}$ & Phase III & $\begin{array}{l}\text { Randomized, } \\
\text { open-label }\end{array}$ & Pancreatic cancer & $\begin{array}{l}\text { mOS: } 6.1 \text { (Onivyde + 5-FU/LV) vs } 4.2(5-\mathrm{FU} / \mathrm{LV}) \text { months } \\
\text { mPFS: } 3.1 \text { (Onivyde + 5-FU/LV) vs I.5 (5-FU/LV) months }\end{array}$ \\
\hline
\end{tabular}

Abbreviations: MTD, maximum tolerated dose; ORR, objective response rate; 5-FU/LV, 5-fluorouracil/leucovorin; mOS, median overall survival; mPFS, median progressionfree survival. 
profile, pharmacokinetic variables, and antitumor activity of Onivyde. ${ }^{22}$ The MTD of Onivyde monotherapy at 3-week interval was $120 \mathrm{mg} / \mathrm{m}^{2}$, which was the recommended dose for future studies. Myelosuppression and diarrhea were the major DLTs. The toxicity pattern was comparable with that of free-form irinotecan, and the most common toxicity observed in the six patients at the MTD dose level $\left(120 \mathrm{mg} / \mathrm{m}^{2}\right)$ was diarrhea (100\% in all grades, $33 \%$ in grade $3-4)$ and vomiting (83.3\% in all grades, $66.7 \%$ in grade $3-4)$. In addition, promising antitumor activities from Onivyde were observed in the patients who were refractory to currently available treatments. Among them, two patients achieved partial response, one with pancreatic cancer who failed several lines of treatment including gemcitabine and 5-fluorouracil/leucovorin (5-FU/LV) alone or in combination with oxaliplatin, and the other with cervical cancer whose tumor relapsed after cisplatin-based concurrent chemoradiotherapy. Another three patients with breast cancer, pancreatic neuroendocrine tumor, and thymic carcinoma had stable disease. The disease control rate was $45.5 \%$ for intent-to-treat patients.

\section{Phase II}

\section{Onivyde for locally advanced or metastatic gastric or OG junction adenocarcinoma}

OG cancer represents a significant global health problem with an estimated one million cases diagnosed every year in the world..$^{18}$ There may be clinical remission or disease stabilization in advanced OG cancer patients who receive first-line chemotherapy; however, most will ultimately experience disease progression, and thus, effective secondline chemotherapy is essential. ${ }^{24}$ However, currently, there are no standard second-line treatments in this setting..$^{25,26}$ Therefore, novel and more effective treatment options are urgently needed to provide survival benefit for patients with advanced OG cancer. Fortunately, the novel liposomal nanocarrier formulation of irinotecan, Onivyde, is a new therapy option providing hope for patients with advanced OG cancer who failed one prior chemotherapy regimen. In a randomized three-arm Phase II study (NCT00813072), Roy et al evaluated the efficacy and safety of single-agent Onivyde compared with irinotecan or docetaxel in the second-line treatment of advanced OG cancer. ${ }^{18}$ In this trial, patients with locally advanced/metastatic OG cancer who had failed one prior chemotherapy regimen were randomly divided into three groups: Onivyde $120 \mathrm{mg} / \mathrm{m}^{2}$, irinotecan $300 \mathrm{mg} / \mathrm{m}^{2}$, and docetaxel $75 \mathrm{mg} / \mathrm{m}^{2}$ every 3 weeks. The primary end point was objective response rate (ORR). The secondary end points included progression-free survival (PFS), overall survival (OS), and 1-year survival rate. The outcomes demonstrated that the ORR for Onivyde, irinotecan, and docetaxel was $13.6 \%(6 / 44 ; 95 \%$ confidence interval [CI]: 5.2-27.4), 6.8\% (3/44; 95\% CI: $1.4-18.7)$, and $15.9 \%$ (7/44; 95\% CI: 6.6-30.1), respectively. The ORR associated with Onivyde was comparable with docetaxel and numerically greater than that of irinotecan. The median overall survival (mOS) and median progression-free survival (mPFS) were similar in all the three arms. The mOS for Onivyde, irinotecan, and docetaxel was 7.3 (95\% CI: 3.84-9.17), 7.8 (95\% CI: 4.90-9.20), and 7.7 months (95\% CI: 5.32-12.32), respectively. The mPFS was 2.7 (95\% CI: 1.54-3.65), 2.6 (95\% CI: 1.48-4.34), and 2.7 months (95\% CI: 1.41-5.45), respectively. Kaplan-Meier estimates of 1-year survival rates were $21.3 \%, 30.8 \%$, and $40.4 \%$, respectively, in those three treatment arms. Treatment was well tolerated, and Onivyde was associated with an increased frequency of grade 3-4 diarrhea (27.3\% in Onivyde group vs $18.2 \%$ in irinotecan group) and nausea, with similar rates of vomiting, neutropenia, and febrile neutropenia compared with irinotecan and docetaxel. These results illustrated that Onivyde is well tolerated and also has a comparable efficacy to docetaxel and irinotecan in patients with locally advanced or metastatic OG cancer, who failed first-line treatment.

\section{Onivyde for $\mathrm{mCRC}$}

Colorectal cancer ranks as the third most common cancer worldwide as approximately one million new cases are diagnosed annually., ${ }^{3,27,28}$ Chemotherapeutic agents are widely used for patients with mCRC to prolong survival, control symptoms, and improve the quality of life. Irinotecan is one of the key drugs used for the management of mCRC, along with oxaliplatin. ${ }^{28}$ These drugs are often combined with 5-FU/LV in regimens such as FOLFIRI (irinotecan, 5-FU, and LV) or FOLFOX (oxaliplatin, 5-FU, and LV), which have exhibited clinical effects and considerably improved OS in patients with mCRC in recent decades. ${ }^{3,27,28}$ In a randomized noncomparative Phase II study (NCT01375816), Chibaudel et al evaluated Onivyde or irinotecan in combination with 5-FU/LV as secondline therapy in patients with $\mathrm{mCRC}$ who had failed one prior oxaliplatin-based first-line therapy. ${ }^{19}$ In the trial, 55 patients were randomly assigned to FUPEP $(\mathrm{n}=28$, Onivyde +5 -FU/LV) or FOLFIRI ( $\mathrm{n}=27$, irinotecan +5 -FU/LV). Bevacizumab q2w $(5 \mathrm{mg} / \mathrm{kg})$ was allowed in both arms. The primary end point was ORR. The outcomes demonstrated that in the evaluable population $(\mathrm{n}=50)$, ORR was $16.7 \%(\mathrm{n}=4 / 24)$ and $11.5 \%$ $(n=3 / 26)$ in the FUPEP and FOLFIRI arms, respectively. Most common grade 3-4 adverse events reported in the respective 
FUPEP and FOLFIRI arms were diarrhea (21\% vs 33\%), neutropenia (11\% vs $30 \%)$, mucositis ( $11 \%$ vs $11 \%)$, and alopecia (G2: $25 \%$ vs 26\%). Hence, Onivyde may provide a new second-line treatment option for $\mathrm{mCRC}$.

\section{Onivyde for gemcitabine-refractory metastatic pancreatic cancer}

Pancreatic cancer is well recognized as an extremely challenging disease on multiple fronts, which is characterized by late detection, poor prognosis, and aggressive metastasis. ${ }^{29,30}$ Early detection is uncommon with no $>15 \%-20 \%$ of the patients being amenable for curative intent surgery at the time of diagnosis. ${ }^{12}$ Even in these cases, 5-year overall survival is still only $22 \%$. Systemic chemotherapy based on gemcitabine is the popular regimen for patients with advanced pancreatic cancer, the mOS of whom is generally $\sim 6$ months. ${ }^{12,31}$ For pancreatic cancer, irinotecan has not been considered as a clinically useful drug. However, since Conroy et $\mathrm{al}^{32}$ demonstrated that FOLFIRINOX regimen consisting of oxaliplatin, irinotecan, and 5-FU/LV could provide significantly better ORR, PFS, and OS than gemcitabine monotherapy in patients with metastatic pancreatic cancer, irinotecan-included therapy has been receiving increased attention for advanced pancreatic cancer. ${ }^{33}$ However, notably and not unexpectedly, this triplet regimen is often hindered by the significant toxicity. Fortunately, the novel nanoliposomal formulation of irinotecan, Onivyde, could present safe therapeutic option. In an international, multicenter, open-label, Phase II trial (NCT00813163), Ko et al evaluated Onivyde monotherapy as second-line treatment for patients with gemcitabine-based chemotherapy-resistant metastatic pancreatic cancer. ${ }^{20}$ In the study, a total of 40 patients were enrolled. The primary end point of this trial was 3-month survival rate $\left(\mathrm{OS}_{3 \text {-month }}\right)$. Secondary end points included ORR, PFS, clinical benefit response, CA19-9 tumor marker response, and safety profile. Of the 40 treated patients, three patients $(7.5 \%)$ achieved an objective response, with an additional 17 (42.5\%) demonstrating stable disease for a minimum of two cycles. Ten (31.3\%) out of 32 patients with an elevated baseline CA19-9 had a $45 \%$ biomarker decline. The study met its primary end point with an $\mathrm{OS}_{3 \text {-month }}$ of $75 \%$ and $\mathrm{mOS}$ and $\mathrm{mPFS}$ of 5.2 and 2.4 months, respectively. The most common severe adverse events included neutropenia (40\%), abdominal pain (37.5\%), and diarrhea (75\%). These results illustrated that Onivyde showed moderate antitumor activity with a manageable side effect profile in patients with metastatic, gemcitabinerefractory pancreatic cancer.

\section{Phase III}

As illustrated, Onivyde provides a new treatment option and a new hope for patients with gemcitabine-based chemotherapyresistant metastatic pancreatic cancer. Further study was carried out. A randomized, open-label, Phase III trial by Von Hoff et al investigated Onivyde, with or without 5-FU/LV, vs 5 -FU/LV, in patients with metastatic pancreatic cancer progressed on or following gemcitabine-based therapy (NCT01494506). ${ }^{21}$ Patients with metastatic pancreatic cancer after gemcitabine-based therapy were randomized 1:1:1 to receive (A) Onivyde, (B) 5-FU/LV, or (C) combination of Onivyde and 5-FU/LV. The primary end point was OS in arms $\mathrm{A}$ and $\mathrm{C}$, each vs the control arm B. The outcomes demonstrated that OS, PFS, ORR, and time to failure were significantly improved by Onivyde +5 -FU/LV compared with 5-FU/LV. mOS was 6.1 months (95\% CI: 4.8-8.9) in the Onivyde + 5-FU/LV arm and 4.2 months (95\% CI: 3.3-5.3) in the $5-\mathrm{FU} / \mathrm{LV}$ arm (hazard ratio $[\mathrm{HR}]=0.67, P=0.012$ ). $\mathrm{mPFS}$ was 3.1 (95\% CI: $2.7-4.2)$ and 1.5 months (95\% CI: 1.4-1.8), respectively $(\mathrm{HR}=0.56, P<0.001)$. Onivyde alone did not demonstrate a statistical improvement in efficacy compared with $5-\mathrm{FU} / \mathrm{LV}$. The most common grade $>3$ toxicities seen with the combination of Onivyde and 5-FU/LV were neutropenia (14.5\%), fatigue (13.7\%), diarrhea (12.8\%), and vomiting (11.1\%). Given the excellent results obtained in the Phase III trial, the FDA newly approved Onivyde + 5-FU/LV regimen for patients with gemcitabine-based chemotherapyresistant metastatic pancreatic cancer. An updated analysis of OS, 6- and 12-month survival estimates, and safety was presented in the 2016 Gastrointestinal Cancers Symposium, San Francisco, CA, USA. Onivyde + 5-FU/LV $(n=117)$ retained an OS advantage relative to 5-FU/LV ( $\mathrm{n}=119)$ : 6.2 (95\% CI: 4.8-8.4) vs 4.2 months (95\% CI: 3.3-5.3) with an unstratified HR of $0.75(P=0.0417)$. Six-month survival estimates were 53\% (95\% CI: 44\%-62\%) for Onivyde + 5-FU/ LV vs 38\% (95\% CI: 29\%-47\%) for 5-FU/LV; 12-month survival estimates were 26\% (95\% CI: 18\%-35\%) for Onivyde + 5-FU/LV vs $16 \%$ (95\% CI: 10\%-24\%) for 5-FU/LV. ${ }^{34}$

\section{Ongoing trials}

Currently, there are also multiple ongoing clinical trials investigating antitumor efficiency of Onivyde. For example, a Phase I study which explores Onivyde + cyclophosphamide in pediatric solid tumors is underway in the South Plains Oncology Consortium (NCT02013336). A randomized, open-label, Phase II study of Onivyde-containing regimens vs nab-Paclitaxel + gemcitabine in patients with previously untreated, metastatic pancreatic adenocarcinoma is actively 
recruiting (NCT02551991). A study of liposomal irinotecan and veliparib in treating patients with solid tumors that are metastatic or cannot be removed by surgery is yet to recruit (NCT02631733). A dose escalation study of MM-398 + irinotecan in patients with unresectable advanced cancer is also recruiting (NCT02640365). Activity of MM-398, a nanoliposomal irinotecan (nal-IRI), in Ewing's family tumor xenografts has been found associated with high exposure of tumor to drug and high SLFN11 expression, which improves antitumor activity compared with the current clinical formulation of irinotecan. ${ }^{8}$ Other liposomal formulations of irinotecan or SN-38 have also been developed, such as IHL-305 (pegylated liposomal irinotecan) and LE-SN-38 (a liposome-encapsulated SN-38). But PEP02 at $120 \mathrm{mg} / \mathrm{m}^{2}$ showed higher SN-38 exposure than IHL-305 at $160 \mathrm{mg} / \mathrm{m}^{2}$. Notwithstanding the relatively high SN-38 AUC, unfortunately, LE-SN-38 did not meet the prespecified activity criteria in the Phase II CALGB 80402 study on mCRC patients. ${ }^{22}$

\section{Side effects of Onivyde treatment}

Onivyde is generally well tolerated by patients. However, adverse effects also occur, and they are considered manageable. The most frequently observed drug-related adverse events were diarrhea, myelosuppression, vomiting, abdominal pain, asthenia, mucositis, and alopecia. ${ }^{18-22}$ Management of these toxicities could include antidiarrheal agents, anticholinergic agents, and dose reduction, interruption, or termination.

\section{Conclusion and future directions for drug development}

The potential advantages of Onivyde, a novel nanoliposomal formulation of irinotecan, include improvement of pharmacokinetics and biodistribution, extension of the circulation time, increase in passive targeting and tumor accumulation via EPR effect, and reduction in organ toxicity. Onivyde as a promising agent has demonstrated improved outcomes and a tolerable safety profile in the treatment of a variety of tumor types. Since single anticancer agent has not always been sufficiently effective, the combination of Onivyde with conventional cytotoxic agent and/or recently emergent moleculartargeted agent should also be investigated to improve the clinical outcomes. Moreover, the optimal dosages of Onivyde for such combinations remain to be determined. Besides, predictive biomarkers could identify cancer indications and patient populations with an increased likelihood of Onivyde responsiveness and avoid exposure to useless toxic medicine.
Thus, additional studies into biomarkers may be useful in predicting personalized therapeutic response.

\section{Acknowledgment}

This work was supported by the National Natural Science Foundation of China (81371678).

\section{Disclosure}

The author reports no conflicts of interest in this work.

\section{References}

1. Xiao XG, Xia S, Zou M, et al. The relationship between UGT1A1 gene polymorphism and irinotecan effect on extensive-stage small-cell lung cancer. Onco Targets Ther. 2015;8:3575-3583.

2. Liu Y, Luan L, Wang X. A randomized Phase II clinical study of combining panitumumab and bevacizumab, plus irinotecan, 5-fluorouracil, and leucovorin (FOLFIRI) compared with FOLFIRI alone as secondline treatment for patients with metastatic colorectal cancer and KRAS mutation. Onco Targets Ther. 2015;8:1061-1068.

3. Suenaga M, Mizunuma N, Matsusaka S, et al. Phase II study of reintroduction of oxaliplatin for advanced colorectal cancer in patients previously treated with oxaliplatin and irinotecan: RE-OPEN study. Drug Des Devel Ther. 2015;9:3099-3108.

4. Ryuge S, Jiang SX, Wada M, et al. Long-term disease-free survivor of metastatic large-cell neuroendocrine carcinoma of the lung treated with amrubicin and irinotecan. Drug Des Devel Ther. 2009;3:213-217.

5. Garcia-Carbonero R, Supko JG. Current perspectives on the clinical experience, pharmacology, and continued development of the camptothecins. Clin Cancer Res. 2002;8(3):641-661.

6. Mathijssen RH, van Alphen RJ, Verweij J, et al. Clinical pharmacokinetics and metabolism of irinotecan (CPT-11). Clin Cancer Res. 2001; 7(8):2182-2194.

7. Drummond DC, Noble CO, Guo Z, Hong K, Park JW, Kirpotin DB. Development of a highly active nanoliposomal irinotecan using a novel intraliposomal stabilization strategy. Cancer Res. 2006;66(6):3271-3277.

8. Kang MH, Wang J, Makena MR, et al. Activity of MM-398, nanoliposomal irinotecan (nal-IRI), in Ewing's family tumor xenografts is associated with high exposure of tumor to drug and high SLFN11 expression. Clin Cancer Res. 2015;21(5):1139-1150.

9. Rivory LP. Metabolism of CPT-11. Impact on activity. Ann N Y Acad Sci. 2000;922:205-215.

10. Etienne-Grimaldi MC, Boyer JC, Thomas F, et al. UGT1A1 genotype and irinotecan therapy: general review and implementation in routine practice. Fundam Clin Pharmacol. 2015;29(3):219-237.

11. Akimoto K, Kawai A, Ohya K. Kinetic-studies of the hydrolysis and lactonization of camptothecin and its derivatives, Cpt-11 and Sn-38, in aqueous-solution. Chem Pharm Bull (Tokyo). 1994;42:2135-2138.

12. Tsai CS, Park JW, Chen LT. Nanovector-based therapies in advanced pancreatic cancer. J Gastrointest Oncol. 2011;2(3):185-194.

13. Zhang $\mathrm{H}$. Multifunctional nanomedicine platforms for cancer therapy. J Nanosci Nanotechnol. 2012;12(5):4012-4018.

14. Zhang H, Wang C, Chen B, Wang X. Daunorubicin-TiO2 nanocomposites as a "smart" pH-responsive drug delivery system. Int J Nanomedicine. 2012;7:235-242.

15. Zhang H, Xiong J, Guo L, Patela N, Guang X. Integrated traditional Chinese and western medicine modulator for overcoming the multidrug resistance with carbon nanotubes. RSC Adv. 2015;5:71287-71296.

16. Zhang H, Patela N, Xiong J, Ding S. Targeting and noninvasive treatment of hepatocellular carcinoma in situ by $\mathrm{ZnO}$ nanorod-mediated concurrent chemoradiotherapy. RSC Adv. 2015;5:85720-85729.

17. Tahover E, Patil YP, Gabizon AA. Emerging delivery systems to reduce doxorubicin cardiotoxicity and improve therapeutic index: focus on liposomes. Anticancer Drugs. 2015;26(3):241-258. 
18. Roy AC, Park SR, Cunningham D, et al. A randomized phase II study of PEP02 (MM-398), irinotecan or docetaxel as a second-line therapy in patients with locally advanced or metastatic gastric or gastro-oesophageal junction adenocarcinoma. Ann Oncol. 2013;24(6): 1567-1573.

19. Chibaudel B, Maindrault-Goebel F, André T, et al. PEPCOL: a randomized noncomparative phase II study of PEP02 (MM-398) or irinotecan in combination with leucovorin and 5-fluorouracil as second-line therapy in patients with unresectable metastatic colorectal cancer - a GERCOR Study. J Clin Oncol. 2015;33(Suppl 3):Abstr 751.

20. Ko AH, Tempero MA, Shan YS, et al. A multinational phase 2 study of nanoliposomal irinotecan sucrosofate (PEP02, MM-398) for patients with gemcitabine-refractory metastatic pancreatic cancer. Br J Cancer. 2013;109(4):920-925.

21. Von Hoff D, Li CP, Wang-Gillam A, et al. Napoli-1: randomized phase 3 study MM-398 (nal-IRI), with or without 5-fluorouracil and leucovorin, versus 5-fluorouracil and leucovorin, in metastatic pancreatic cancer progressed on or following gemcitabine-based therapy. Ann Oncol. 2014;25(Suppl 2):ii105-ii106.

22. Chang TC, Shiah HS, Yang CH, et al. Phase I study of nanoliposomal irinotecan (PEP02) in advanced solid tumor patients. Cancer Chemother Pharmacol. 2015;75(3):579-586.

23. Kalra AV, Kim J, Klinz SG, et al. Preclinical activity of nanoliposomal irinotecan is governed by tumor deposition and intratumor prodrug conversion. Cancer Res. 2014;74(23):7003-7013.

24. Zhang H. Apatinib for molecular targeted therapy in tumor. Drug Des Devel Ther. 2015;9:6075-6081.

25. Wesolowski R, Lee C, Kim R. Is there a role for second-line chemotherapy in advanced gastric cancer? Lancet Oncol. 2009;10(9):903-912.

26. Lordick F. Gastrointestinal cancer: salvage chemotherapy in gastric cancer - more than a straw? Nat Rev Clin Oncol. 2012;9(6):312-313.
27. Liang RF, Zheng LL. The efficacy and safety of panitumumab in the treatment of patients with metastatic colorectal cancer: a meta-analysis from five randomized controlled trials. Drug Des Devel Ther. 2015; 9:4471-4478.

28. Suenaga M, Mizunuma N, Matsusaka S, et al. A phase I/II study of biweekly capecitabine and irinotecan plus bevacizumab as second-line chemotherapy in patients with metastatic colorectal cancer. Drug Des Devel Ther. 2015;9:1653-1662.

29. Wang C, Zhang H, Chen Y, Shi F, Chen B. Gambogic acid-loaded magnetic $\mathrm{Fe}(3) \mathrm{O}(4)$ nanoparticles inhibit Panc-1 pancreatic cancer cell proliferation and migration by inactivating transcription factor ETS1. Int J Nanomedicine. 2012;7:781-787.

30. Wang C, Zhang H, Chen B, Yin H, Wang W. Study of the enhanced anticancer efficacy of gambogic acid on Capan-1 pancreatic cancer cells when mediated via magnetic Fe3O4 nanoparticles. Int J Nanomedicine. 2011;6:1929-1935.

31. Moore MJ, Goldstein D, Hamm J, et al. Erlotinib plus gemcitabine compared with gemcitabine alone in patients with advanced pancreatic cancer: a phase III trial of the National Cancer Institute of Canada Clinical Trials Group. J Clin Oncol. 2007;25(15):1960-1966.

32. Conroy T, Desseigne F, Ychou M, et al. FOLFIRINOX versus gemcitabine for metastatic pancreatic cancer. $N$ Engl J Med. 2011;364(19): 1817-1825.

33. Vaccaro V, Sperduti I, Milella M. FOLFIRINOX versus gemcitabine for metastatic pancreatic cancer. N Engl J Med. 2011;365(8):768-769.

34. Wang-Gillam A, Li CP, Bodoky G, et al. Phase III study of nanoliposomal irinotecan (nal-IRI, MM-398), with or without 5-fluorouracil and leucovorin (5-FU/LV), versus 5-FU/LV in metastatic pancreatic cancer (mPAC) previously treated with gemcitabine-based therapy. J Clin Oncol. 2016;34(Suppl 4S):Abstr 417.
OncoTargets and Therapy

\section{Publish your work in this journal}

OncoTargets and Therapy is an international, peer-reviewed, open access journal focusing on the pathological basis of all cancers, potential targets for therapy and treatment protocols employed to improve the management of cancer patients. The journal also focuses on the impact of management programs and new therapeutic agents and protocols on

\section{Dovepress}

patient perspectives such as quality of life, adherence and satisfaction. The manuscript management system is completely online and includes a very quick and fair peer-review system, which is all easy to use. Visit http://www.dovepress.com/testimonials.php to read real quotes from published authors. 